\title{
Organization Role
}

National Cancer Institute

\section{Source}

National Cancer Institute. Organization Role. NCI Thesaurus. Code C114551.

A title that describes the function of a designated group, governing body, or institution. 